\title{
Atomic Layer Deposition of the Lithium-Silicon-Tin Oxide System for Solid-State Thin-Film Lithium Batteries
}

\author{
I. V. Mitrofanov ${ }^{a}$, D. S. Nazarov ${ }^{a}$, A. A. Popovich ${ }^{a}$, and M. Yu. Maximov ${ }^{a}$ * \\ ${ }^{a}$ Peter the Great St. Petersburg Polytechnic University, Petersburg, Russia \\ *e-mail:maximspbstu@mail.ru \\ Received June 3, 2019; revised July 16, 2019; accepted July 17, 2019
}

\begin{abstract}
Materials and methods for creating the elements of thin-film lithium-ion batteries (TFLBs) are considered.
\end{abstract}

Keywords: lithium-ion battery, lithium oxide, anode, lithiated tin oxide, thin-film electrode, atomic layer deposition, molecular layering

DOI: $10.1134 / \mathrm{S} 003602952013025 \mathrm{X}$

The development of energy storage materials is one of the most important problems of modern materials science [1, 2]. Solid-state thin-film lithium-ion batteries (TFLBs) are widely used in microelectronics, stand-alone sensors, data storage devices, implants, and so on [2]. Thus, the development of new materials to improve the functional characteristics of TFLBs is a challenging problem of modern science and industry.

Tin oxide is one of the most promising materials for the anode of lithium ion batteries due to its high theoretical and practical specific capacitance [2]: for metallic tin, the specific capacitance is $991 \mathrm{~mA} \mathrm{~h} / \mathrm{g}$. Thus, $\mathrm{Sn} / \mathrm{SnO}_{2}$-based anode materials have a specific capacitance of 800-1000 mA h/g [2].

The electrochemical characteristics of anodes depend strongly on their manufacturing technology. Among the various technological methods, atomic layer deposition (ALD, molecular layering-ML)) and should be noted. ALD makes it possible to manufacture thin-film electrodes of various chemical compositions $[1,2]$ with excellent conformity, uniformity, and thickness control [2].

Lithium oxide-silicon-tin nanofilms were synthesized by ALD using lithium bis(trimethylsilyl)amide (LiHMDS) and tetraethyltin (TET) as metal-containing reagents and oxygen plasma, ozone, and water as co-reagents. Single-crystal (100) silicon and 316SS stainless steel were used as substrates. The nanofilm thickness was determined by spectral ellipsometry and scanning electron microscopy. The experimental results demonstrate that oxygen plasma is the optimum co-reagents. Composition and structure were studied by secondary ion mass spectroscopy, $\mathrm{X}$-ray photoelectron spectroscopy, and X-ray diffraction. The nanofilms contained silicon as an impurity, the source of which was likely to be reagent LiHMDS. The stainless steel nanofilms demonstrated a high Coulomb efficiency (99.1-99.8\%) and cyclic characteristics at a voltage of $0.01-2.00 \mathrm{~V}$.

\section{FUNDING}

The research was supported by the Ministry of Science and Higher Education of the Russian Federation (State Assignment for basic research 0784-2020-0022).

\section{REFERENCES}

1. M. Maximov, P. Novikov, A. Rymyantsev, Y. Koshtyal, D. Nazarov, A. Silin, and A. Popovich, "Application of atomic layer deposition in Li-ion batteries," in Proceedings of 8th International Conference on Nanomaterials NANOCON 2016 (2016), pp. 296-302.

2. M. Maximov, D. Nazarov, I. Mitrofanov, Y. Koshtyal, A. Rymyantsev, and A. Popovich, "Atomic layer deposition of lithium oxide, tin oxide, and lithiated tin oxide nanofilms for high performance thin film batteries anodes," in Proceedings of 10th International Conference on Nanomaterials NANOCON 2018 (2018), pp 169-174.

Translated by K. Shakhlevich 